\title{
Assessing Oral Medication Adherence and Identifying Predictors of Low Adherence in Chinese Inflammatory Bowel Disease Patients
}

This article was published in the following Dove Press journal: Patient Preference and Adherence

\section{Wen $\mathrm{Hu}^{*}$ \\ Shurong $\mathrm{Hu}^{*}$ \\ Yimiao Zhu \\ Hanwen Chen (D) \\ Yan Chen}

Center for Inflammatory Bowel Diseases, Department of Gastroenterology, The Second Affiliated Hospital, Zhejiang University School of Medicine, Hangzhou 310009, People's Republic of China

*These authors contributed equally to this work
Correspondence: Yan Chen

Tel +86-57l-87783936

Email chenyan72_72@zju.edu.cn
Background: Poor medication adherence in inflammatory bowel disease (IBD) had a negative impact on disease outcomes. In this study, we aimed to determine predictors of low adherence in the Chinese IBD populations and also aimed to compare a self-reported scale to a pharmacy refill index in assessing adherence of 5-ASA and azathioprine taken by Chinese IBD patients.

Patients and Methods: Adult patients with IBD who had been taking 5-ASA or azathioprine for at least 3 months were recruited from hospital outpatient clinics. The MPR was calculated from previous six-month pharmacy refill data and the self-reported Morisky Medication Adherence Scale (MMAS-8) was issued through QR code questionnaires. Intentional and unintentional adherence scores were calculated according to specific items. Non-adherence was defined as MMAS- 8 scores $<6$ or MPR $<0.8$.

Results: The response rate in the IBD patients was as high as $97 \%$. 5-ASA non-adherence rate assessed by MPR was $30 \%$ and $37 \%$ by MMAS- 8 , and azathioprine non-adherence rate assessed was $33 \%$ by both MPR and MMAS-8. In a linear regression analysis, MPR value was significantly correlated with MMAS-8 score in 5-ASA group ( $r=0.4, p=0.003)$, and significantly correlated with unintentional adherence score $(r=0.47, \mathrm{p}<0.001)$. No significant correlation was observed between MPR value and MMAS- 8 score in azathioprine group. Multivariate analysis demonstrated that age (OR: $1.08 ; 95 \% \mathrm{CI}: 1.02-1.13 ; \mathrm{P}=0.0015)$ and previous abdominal surgery (OR: 3.18; 95\% CI: 2.09-4.27; $\mathrm{P}=0.04$ ) were associated with high medication adherence. While patients who had small intestine lesion (OR: 0.09 ; 95\% CI: $0.01-0.17 ; \mathrm{P}=0.006$ ) were associated with low adherence.

Conclusion: Predictors of low adherence were young age, lesions on small intestine, whereas previous abdominal surgery was a protective factor. This study also demonstrated that the MMAS-8 scale was a valid instrument for assessing 5-ASA adherence in IBD patients. Unintentional non-adherence was significantly related to the total non-adherence, which would allow to use the tool to seek ways for adherence improvement.

Keywords: inflammatory bowel disease, medication adherence, self-reported Morisky Medication Adherence Scale, Medication possession ratio

\section{Introduction}

Inflammatory bowel diseases (IBD), including ulcerative colitis (UC) and Crohn's disease (CD), with an incidence rate of 3.44 per 100000 people in China, are chronic relapsing immune-mediated inflammatory conditions which require lifelong treatment. ${ }^{1}$ At present, the main drugs applied in the treatment of IBD are 5-aminosalicylic acid (5-ASA), corticosteroids, immunoregulator (eg azathioprine) and 
biologics (e.g.infliximab). ${ }^{2}$ For many chronic diseases including IBD, adherence to long-term therapies in patients are related to alleviate symptoms, prevent disease progress, decrease disease flares, increase quality of life and decrease societal and personal costs. ${ }^{3-5}$ Previous study showed that non-adherence rates to oral 5-aminosalicylates and thiopurines for IBD are ranging from $7 \%$ to $72 \%$, depending on the tools measured. ${ }^{6}$ And rates of nonadherence to biologic medications for IBD have been estimated from $17 \%$ to $45 \%$ depending on therapy with anti-tumor necrosis factor agents. ${ }^{7}$ Some studies have showed that being single, female, younger age, higher educational level as predictors for low medication adherence. ${ }^{8}$ Others demonstrated that good communication with the physician and understanding the disease as predictors for high adherence. ${ }^{9}$

Different tools such as patient questionnaire, patient diary, pharmacy refill data, interviews, physician perception, pill counters, drug levels and drug metabolites are used to identify and measure adherence. ${ }^{10}$ In general, objective assessments of adherence are lower than subjective measurements. Several questionnaires are validated for use in other chronic diseases such as hypertension and diabetes to measure medication adherence. Among them, the modified Morisky Medication Adherence Scale (MMAS-8), a selfadministered questionnaire, an eight-item screening tool, has been validated to measure IBD therapy adherence and served as a useful tool in IBD. ${ }^{11}$ However, MMAS can only be used under license and the cost is prohibitive for many health services. According to the scale, patients can be divided into low, medium and high adherers. ${ }^{11}$ Besides, the continued use of a medication for the duration prescribed is termed as patient's persistence which is an objective measurement of adherence. Intentional and unintentional nonadherence are two specific dimensions of evaluation and understanding of patients' medication-taking behavior. Medication possession ratio (MPR) is defined as the number of days of supply of drug obtained divided by the number of days in a study period. According to MPR, patients can be divided into non-adherence and adherence.

Data regarding IBD therapy adherence from China are missing. In this context, the primary outcome of interest was to identify predictors of low and high adherence in a cohort of Chinese IBD patients. Additional outcomes of interest included rates of adherence to 5-aminosalicylates and thiopurines, differences in adherence rates between self-administered scale and medication possession ratio (MPR) in our cohort. Finally, we aimed to compare the validity and reliability of MMAS- 8 and MPR in measuring medication adherence in Chinese IBD patients.

\section{Patients and Methods Study Design}

Consecutive patients were recruited in IBD outpatient clinic at the Second Affiliated Hospital, School of Medicine, Zhejiang University, China, between October 2018 and February 2019. Included patients had a diagnosis of IBD according to the usual clinical, endoscopic, radiologic, or pathology criteria. They were invited to complete an online questionnaire app in Chinese. Inclusion criteria included age more than 18 years, current use of self-administered medication to treat IBD and they had a minimum of 6 months' supply refill data. Patients were excluded if they had difficulty in understanding the content. The questionnaires included social demographic data, disease parameters, personal habits and current therapy, as described below. Patients were also invited to fill in the modified Morisky Medication Adherence Scale (MMAS-8) (Table 1), ${ }^{12-14}$ which was an 8-question survey with seven questions having a binary answer (yes/no) and a 5-point Likert response for the last item. Each item of MMAS-8 investigated a specific medication-taking behavior but not a determinant of adherence behavior. In MMAS table, items $1,4,5$ and 8 were relevant with unintentional non-adherence behavior while items 2,3,6,7 were related to intentional non-adherence. Medication possession ratio (MPR) was calculated for each therapy, which was calculated as (sum of the days of medication supplied)/(the sum of the days in the total refill intervals). Through tracking refills over a 6-month period, patients' refill activity was averaged and accounted for premature refills. Pharmacy refills were considered as a suitable gold standard to measure patient medical adherence. ${ }^{15}$ The study was approved by the Ethics Committee of the Second Affiliated Hospital, School of Medicine, Zhejiang University in China. Informed consent was verbally received from all patients who completed the questionnaire. The IRB approved the verbal informed consent process.

\section{Patients' Characteristics}

The social demographic profile included current age, sex, marital status (married, not married, divorced), education level (illiterate, primary school certificate, middle school certificate, high school/secondary vocational school diploma, three-year college diploma, bachelor's certificate, master's and doctorate's certificate), level of income per month (under 650€, 650-1300€, 1300-2600€ and above 
Table I Modified MMAS-8 for IBD Patients and Frequencies for MMAS-8 Scale

\begin{tabular}{|c|c|c|c|}
\hline MMAS Questions & Scoring & 5-ASA & Azathioprine \\
\hline I. Do you sometimes forget to take your IBD medication? & $\begin{array}{l}\text { I No; } \\
0 \text { Yes }\end{array}$ & $55 \%$ & $44 \%$ \\
\hline $\begin{array}{l}\text { 2. People sometimes miss taking their medications for reasons other than forgetting. Thinking over the } \\
\text { past } 2 \text { weeks, was there a time when you did not take your medication? }\end{array}$ & $\begin{array}{l}\text { I No; } \\
0 \text { Yes }\end{array}$ & $11 \%$ & $27 \%$ \\
\hline $\begin{array}{l}\text { 3. Have you ever cut back or stopped taking your medication without telling your doctor because you felt } \\
\text { worse when you took it? }\end{array}$ & $\begin{array}{l}\text { I No; } \\
0 \text { Yes }\end{array}$ & $5 \%$ & $12 \%$ \\
\hline 4. When you travel or leave home, do you sometimes forget to bring along your IBD medication? & $\begin{array}{l}\text { I No; } \\
0 \text { Yes }\end{array}$ & $33 \%$ & $21 \%$ \\
\hline 5. Did you take your IBD medicine yesterday or at the last scheduled dose? & $\begin{array}{l}\text { I No; } \\
0 \text { Yes }\end{array}$ & $16 \%$ & $19 \%$ \\
\hline $\begin{array}{l}\text { 6. When you feel like your IBD symptoms are under control, do you sometimes stop taking your } \\
\text { medication? }\end{array}$ & $\begin{array}{l}\text { I No; } \\
0 \text { Yes }\end{array}$ & $25 \%$ & $13 \%$ \\
\hline $\begin{array}{l}\text { 7. Taking medication regularly is a real inconvenience for some people. Do you ever feel hassled about } \\
\text { sticking to your IBD treatment plan? }\end{array}$ & $\begin{array}{l}\text { I No; } \\
0 \text { Yes }\end{array}$ & $9 \%$ & $4 \%$ \\
\hline 8. How often do you have difficulty remembering to take all your IBD medications? & & & \\
\hline Never/rarely & I & & $43 \%$ \\
\hline Once in a while & 0.75 & & $31 \%$ \\
\hline Sometimes & 0.5 & & $24 \%$ \\
\hline Usually & 0.25 & & $2 \%$ \\
\hline All the time & 0 & & $0 \%$ \\
\hline
\end{tabular}

Notes: Use of the MMAS is protected by US copyright laws. Permission for use is required. Licensure agreement is available from Donald E. Morisky, ScD, ScM, MSPH, Professor, Department of Community Health Sciences, UCLA School of Public Health, 650 Charles E. Young Drive South, Los Angeles, California 90095, USA.

2600€) and smoking status (current smoker, ex-smoker, and nonsmoker). The clinical disease profile consisted of type of disease, age at onset, duration of disease, disease location, disease activity and abdominal surgery in the past. The current medications used for IBD included oral 5-aminosalicylic acid (5-ASA)compounds, 5-ASA enemas and suppositories, steroids, immunosuppressant, monoclonal antibody and enteral nutrition. In our study, the clinical disease activity was assessed by the validated patientbased Harvey-Bradshaw Index (HBI) items for patients with $\mathrm{CD}$, and the Patient-based Simple Clinical Colitis Activity Index (P-SSCAI) for patients with UC. ${ }^{16,17}$ And patient-based $\mathrm{CD}$ activity was indicated as active (HBI score $>4$ ) or as inactive (HBI score $\leq 4) .{ }^{17}$ While patientbased UC activity was rated as active (SCCAI score $\geq 5$ ) or as remission (SCCAI score $<5){ }^{16}$

\section{Adherence Measures}

According to MMAS-8 score, patients were divided into three groups: low adherence was defined as a score of less than 6 points, medium adherence by 6-7 points, and high adherence by 8 points. ${ }^{18}$ Intentional and unintentional non- adherence scores were calculated according to specific items. When analyzing the data, score 6-8 points were combined into a single group labeled as "medium and high adherers". Pharmacy refill scores were dichotomized and used to classify adherence as non-adherence (MPR < $80 \%$ ) based on previously validated criteria. ${ }^{18}$

\section{Statistical Analysis}

Data analyses were performed using IBM SPSS software (version 25.0, Chicago, Illinois, USA). For quantitative variables, mean and standard deviation or inter-quartile range $[\mathrm{IQR}]$ was calculated, and comparison of differences between groups was compared using $t$-tests if normally distributed, or with the wilcoxon test if non-normally distributed. Categorical variables were expressed as frequency or percentages, and differences in frequencies between groups were calculated using $\chi 2$ test or Fisher's exact test. In addition, univariate logistic regression analysis was used to determine characteristics associated with therapy adherence. And multivariate logistic regression was used to assess whether these individual variables were related to outcomes. Finally, Spearman correlation 
analysis was used to assess the association between MMAS-8 and MPR percentage. Statistically significant differences were reported as $\mathrm{p}<0.05$.

\section{Results}

\section{Overall Profiles of Chinese IBD Patients}

One hundred and ninety-four IBD patients filled the questionnaire. The response rate in the IBD patients was as high as $97 \%$. Among them, there were 88 patients lacking six-month pharmacy refill data. Altogether, 106 consecutive patients were enrolled in the study for comparing with MMAS- 8 and MPR data. Table 2 summarizes the main characteristics of our study population: 48 (45\%) had ulcerative colitis, 58 (55\%) had Crohn's disease and 73 patients $(69 \%)$ were men. The current mean age was 33 years and the mean duration of the disease was 4 years. In terms of medical therapy, 54 (51\%) IBD patients were on oral 5-Aminosalicylic acid therapy, $26(24 \%)$ on 5-ASA enemas and suppositories therapy, 54 (51\%) were taking thiopurines therapy, $8(8 \%)$ were taking infliximab therapy, $10(9 \%)$ were taking steroids therapy, $11(10 \%)$ were on thalidomide therapy and 27 (26\%) were on enteral nutrition. Of these, 62 IBD patients were on mono-therapy $(58 \%)$ and $44(42 \%)$ were taking combination therapy with two or more drugs (Table 2).

\section{Medication Adherence According to MMAS-8}

According to the MMAS-8 survey, the median MMAS-8 score was 6.8 (IQR 5.4-7.8), 38 (36\%) patients had a score $<6$ and were defined as low adherers, 47 (44\%) scored $>6$ to 8 were defined as medium adherers, and $21(20 \%)$ patients scored $=8$ were defined as high adherers (Table 1 ). The clinical characteristics of these patients are shown in Table 3. There was no significant difference by smoking status, duration of disease, marital status, education level and monthly income for adherence. In addition, assessed by MMAS-8, 5-ASA non-adherence rate was 37\%, azathioprine non-adherence rate was 33\%. Among the MMAS-8 low adherent patients, $91 \%$ patients had non-persistent medication fill rates (MPR $<0.8$ ), whereas $90 \%$ patients had persistent medication fill rates (MPR $\geq 0.8$ ) among the MMAS- 8 high adherent patients. The differences between these two groups were statistically significant $(\mathrm{p}=0.001)$ (Table 3 ). In addition, multivariate logistic binary regression analysis identified that old age (OR: 1.08 ; 95\% CI: $1.02-$ 1.13; $\mathrm{P}=0.0015)$ was associated with high medication
Table 2 Patient Characteristics $(n=106)$

\begin{tabular}{|c|c|c|}
\hline $\begin{array}{l}\text { Sex, n (\%) } \\
\text { Male } \\
\text { Female }\end{array}$ & $\begin{array}{l}73 \\
33\end{array}$ & $\begin{array}{l}69 \% \\
31 \%\end{array}$ \\
\hline $\begin{array}{l}\text { Disease, } n(\%) \\
\text { UC } \\
\text { CD }\end{array}$ & $\begin{array}{l}48 \\
58\end{array}$ & $\begin{array}{l}45 \% \\
55 \%\end{array}$ \\
\hline $\begin{array}{l}\text { Age, median (range) } \\
\text { Age at diagnosis } \\
\text { Duration of disease (range) }\end{array}$ & $\begin{array}{l}33 \\
4\end{array}$ & $\begin{array}{l}19-52 \\
29 \pm 8 \\
3-7\end{array}$ \\
\hline $\begin{array}{l}\text { Smoking, n (\%) } \\
\text { Never smoked } \\
\text { Currently smoking } \\
\text { Ex-smoker }\end{array}$ & $\begin{array}{l}85 \\
9 \\
12\end{array}$ & $\begin{array}{l}80 \% \\
9 \% \\
11 \%\end{array}$ \\
\hline $\begin{array}{l}\text { Disease localization, } \mathrm{n}(\%) \\
\text { Colon } \\
\text { Small intestine } \\
\text { Both colon and small intestine } \\
\text { Unknown }\end{array}$ & $\begin{array}{l}76 \\
39 \\
13 \\
4\end{array}$ & $\begin{array}{l}72 \% \\
37 \% \\
12 \% \\
4 \%\end{array}$ \\
\hline $\begin{array}{l}\text { Abdominal surgery in the past, } n(\%) \\
\text { NO } \\
\text { YES }\end{array}$ & $\begin{array}{l}72 \\
34\end{array}$ & $\begin{array}{l}68 \% \\
32 \%\end{array}$ \\
\hline $\begin{array}{l}\text { Marital status, } \mathrm{n}(\%) \\
\text { Married } \\
\text { Non-Married } \\
\text { Divorced }\end{array}$ & $\begin{array}{l}68 \\
35 \\
3\end{array}$ & $\begin{array}{l}64 \% \\
33 \% \\
3 \%\end{array}$ \\
\hline $\begin{array}{l}\text { Highest level of education completed, } \mathrm{n}(\%) \\
\text { Illiterate } \\
\text { Primary school certificate } \\
\text { Middle school certificate } \\
\text { High school/Secondary vocational school } \\
\text { Three-year college diploma } \\
\text { Bachelor's level } \\
\text { Master's and Doctorate's level }\end{array}$ & $\begin{array}{l}0 \\
3 \\
9 \\
16 \\
24 \\
43 \\
11\end{array}$ & $\begin{array}{l}0 \% \\
3 \% \\
9 \% \\
15 \% \\
23 \% \\
11 \% \\
10 \%\end{array}$ \\
\hline $\begin{array}{l}\text { Self-reported monthly income, } n \text { (\%) } \\
\text { Under } 650 € \\
650-1300 € \\
1300-2600 € \\
\text { Above } 2600 €\end{array}$ & $\begin{array}{l}26 \\
28 \\
32 \\
20\end{array}$ & $\begin{array}{l}25 \% \\
26 \% \\
30 \% \\
19 \%\end{array}$ \\
\hline $\begin{array}{l}\text { Disease activity, } \mathrm{n}(\%) \\
\text { Active } \\
\text { Inactive }\end{array}$ & $\begin{array}{l}25 \\
81\end{array}$ & $\begin{array}{l}24 \% \\
76 \%\end{array}$ \\
\hline $\begin{array}{l}\text { Medication, } \mathrm{n}(\%) \\
\text { Oral 5-aminosalicylic acid } \\
\text { 5-ASA enemas and suppositories } \\
\text { Immunomodulator } \\
\text { Infliximab } \\
\text { Steroids }\end{array}$ & $\begin{array}{l}54 \\
26 \\
54 \\
8 \\
10\end{array}$ & $\begin{array}{l}51 \% \\
24 \% \\
51 \% \\
8 \% \\
9 \%\end{array}$ \\
\hline
\end{tabular}

(Continued) 
Table 2 (Continued).

\begin{tabular}{|l|l|l|}
\hline Thalidomide & II & $10 \%$ \\
Enteral nutrition & 27 & $26 \%$ \\
\hline IBD treatment & & \\
Monotherapy & 62 & $58 \%$ \\
Combination & 44 & $42 \%$ \\
\hline Dosing schedule & & \\
Once-a -day & 42 & $40 \%$ \\
Twice-a-day & 24 & $23 \%$ \\
Three-times-a-day & 27 & $25 \%$ \\
Four-times-a day & 13 & $12 \%$ \\
\hline MMAS-8 score, median (range) & 6.8 & $5.4-7.8$ \\
Low adherer, $\mathrm{n}(\%)$ & 38 & $36 \%$ \\
Medium adherer, $\mathrm{n}(\%)$ & 47 & $44 \%$ \\
High adherer, $\mathrm{n}$ (\%) & 21 & $20 \%$ \\
\hline
\end{tabular}

adherence and additional confirmed that age at diagnosis late (OR: 1.08 ; 95\% CI: $1.01-1.14 ; \mathrm{P}=0.02$ ) was also associated with high adherence (Table 5).

\section{Medication Adherence According to MPR}

When assessing non-adherence by MPR score $<0.8,34$ (32\%)patients were classified as non-adherers. The clinical profiles of these patients are shown in Table 4. There was also no significant difference by smoking status, duration of disease, marital status, education and monthly income for non-adherence. In terms of drug therapy, 5-ASA nonadherence rate assessed by MPR was $30 \%$, while azathioprine non-adherence rate was $33 \%$ (Table 4). Among the MPR non-adherent patients, $82 \%$ of patients were low adherence according to MMAS- 8 . Furthermore, $96 \%$ of patients were high adherence depended on MMAS-8 in the MPR adherent patients. The differences between these two groups were statistically significant $(\mathrm{p}=$ 0.001) (Table 4).

Univariate analysis showed that patients who have small intestine lesion were associated with MPR nonadherence $(\mathrm{P}=0.016)$ (Table 4$)$. Multivariate logistic binary regression analysis identified that previous abdominal surgery (OR: 3.18; 95\% CI: 2.09-4.27; $\mathrm{P}=0.04$ ) was associated with high medication adherence and additional confirmed that patients who had small intestine lesion (OR: 0.09 ; 95\% CI: $0.01-0.17$; $\mathrm{P}=0.006$ ) was associated with low adherence (Table 5).

Besides, in a linear regression analysis, the values of MPR percentage were significantly correlated with MMAS-8 scores in 5-ASA group $(\mathrm{r}=0.4, \mathrm{p}=0.003)$
(Figure 1), and also significantly correlated with unintentional adherence score $(\mathrm{r}=0.47, \mathrm{p}<0.001)$ (Figure 2). However, no significant correlation was observed between MPR value and MMAS-8 score in azathioprine group (data not shown).

\section{Discussion}

Long-term adherence was generally considered as a problem in patients with chronic diseases that needed medication and lifestyle modifications. Adherence refers to the extent of taking medications as prescribed. ${ }^{10}$ Nonadherence in IBD can lead to disease flare, increased hospitalization rate, poor prognosis and other undesirable consequences. However, few studies have been done to observe the problem of Chinese IBD patients' medication adherence. In our study, we used MMAS- 8 scale and MPR to assess patients' medication adherence, and found that almost one third of Chinese IBD patients were low adherent by both MMAS and MPR, which was similar to the proportion of adherence in other countries (35\% to $72 \%)^{8,10,18,19}$ Although different countries had varying health systems, social demographics, access to medication, cost issues, they all had an influence on medication adherence. And low adherence of IBD patients was universal. Therefore, IBD patients' medication adherence was an issue that needed global attention. Approaches such as individualized treatment, self-management programs andemail reminders played a significant role in improving patients' medication adherence. ${ }^{11,20}$

In our study, 5-ASA non-adherence rate was 37\% assessed by MMAS- 8 tool and 30\% assessed by MPR, azathioprine non-adherence rate was $33 \%$ both by MMAS8 tool and MPR, which were similar to those reported in previous studies with the rates of non-adherence from $7 \%$ to $72 \%$, and mostly between $30 \%$ and $45 \%$. ${ }^{6,10}$ Previous studies had showed that being single, active smoking, higher educational level, professional occupation, upper socioeconomic status, and 5-ASA use predicted of low adherence while being self-employed was a protective factor in IBD patients. ${ }^{5,8,21}$ Our study found no correlation between marital status, socioeconomic status, education level, tobacco use and medication adherence. One possible explanation was the existence of cultural differences between China and other countries. In our study, the prescription of different IBD medications at the same time did not seem to affect adherence, and no associations were noticed regarding gender, type of disease, duration of disease, or disease activity. Young age had been identified 
Table 3 Predictive Factors for Adherence According to MMAS (Univariate Analysis)

\begin{tabular}{|c|c|c|c|}
\hline & $\begin{array}{l}\text { Low } \\
\text { Adherence }\end{array}$ & $\begin{array}{l}\text { High } \\
\text { Adherence }\end{array}$ & P-value \\
\hline Patients & $38(36 \%)$ & $68(64 \%)$ & \\
\hline $\begin{array}{l}\text { Gender } \\
\text { Male } \\
\text { Female }\end{array}$ & $\begin{array}{l}25(34 \%) \\
13(39 \%)\end{array}$ & $\begin{array}{l}48(66 \%) \\
20(61 \%)\end{array}$ & 0.609 \\
\hline $\begin{array}{l}\text { Current age (years) } \\
\text { Age at diagnosis } \\
\text { Duration of disease (years) }\end{array}$ & $\begin{array}{l}33(29-36) \\
28 \pm 7 \\
4(3-6)\end{array}$ & $\begin{array}{l}34(29-39) \\
29 \pm 8 \\
4(3-7)\end{array}$ & $\begin{array}{l}0.42 \\
0.62 \\
0.53\end{array}$ \\
\hline $\begin{array}{l}\text { Currently smoking, } n(\%) \\
\text { NO } \\
\text { YES }\end{array}$ & $\begin{array}{l}35(36 \%) \\
3(33 \%)\end{array}$ & $\begin{array}{l}62(64 \%) \\
6(67 \%)\end{array}$ & 0.869 \\
\hline $\begin{array}{l}\text { Abdominal surgery in the past, } \\
\text { n (\%) } \\
\text { NO } \\
\text { YES }\end{array}$ & $\begin{array}{l}28(39 \%) \\
10(29 \%)\end{array}$ & $\begin{array}{l}44(61 \%) \\
24(71 \%)\end{array}$ & 0.34 \\
\hline Disease localization, n (\%) & & & \\
\hline $\begin{array}{l}\text { Colon } \\
\text { NO } \\
\text { YES }\end{array}$ & $\begin{array}{l}12(40 \%) \\
26(34 \%)\end{array}$ & $\begin{array}{l}18(60 \%) \\
50(66 \%)\end{array}$ & 0.366 \\
\hline $\begin{array}{l}\text { Small intestine } \\
\text { NO } \\
\text { YES }\end{array}$ & $\begin{array}{l}21(31 \%) \\
17(44 \%)\end{array}$ & $\begin{array}{l}46(69 \%) \\
22(56 \%)\end{array}$ & 0.145 \\
\hline $\begin{array}{l}\text { Both colon and small intestine } \\
\text { NO } \\
\text { YES }\end{array}$ & $\begin{array}{l}32(34 \%) \\
6(46 \%)\end{array}$ & $\begin{array}{l}61(66 \%) \\
7(54 \%)\end{array}$ & 0.408 \\
\hline $\begin{array}{l}\text { Marital status, n (\%) } \\
\text { Married } \\
\text { Non-Married }\end{array}$ & $\begin{array}{l}23(34 \%) \\
15(39 \%)\end{array}$ & $\begin{array}{l}45(66 \%) \\
23(61 \%)\end{array}$ & 0.561 \\
\hline $\begin{array}{l}\text { Highest level of education } \\
\text { completed, } n(\%) \\
\text { No } \\
\text { YES }\end{array}$ & $\begin{array}{l}18(35 \%) \\
20(37 \%)\end{array}$ & $\begin{array}{l}34(65 \%) \\
34(63 \%)\end{array}$ & 0.477 \\
\hline $\begin{array}{l}\text { Self-reported monthly income, } \\
\text { n (\%) } \\
\text { Under } 5000 ¥ / 5000-10,000 ¥ \\
10,000-20,000 ¥ / \text { above } 20,000 ¥\end{array}$ & $\begin{array}{l}20(37 \%) \\
18(35 \%)\end{array}$ & $\begin{array}{l}34(63 \%) \\
34(65 \%)\end{array}$ & 0.841 \\
\hline \multicolumn{4}{|l|}{$\begin{array}{l}\text { Medication use at } 3 \text { mo of } \\
\text { follow-up, n (\%) }\end{array}$} \\
\hline $\begin{array}{l}\text { 5-ASA use } \\
\text { NO } \\
\text { YES }\end{array}$ & $\begin{array}{l}17(35 \%) \\
21(37 \%)\end{array}$ & $\begin{array}{l}32(65 \%) \\
36(63 \%)\end{array}$ & 0.818 \\
\hline $\begin{array}{l}\text { Thiopurines } \\
\text { NO } \\
\text { YES }\end{array}$ & $\begin{array}{l}20(38 \%) \\
18(33 \%)\end{array}$ & $\begin{array}{l}32(62 \%) \\
36(67 \%)\end{array}$ & 0.582 \\
\hline
\end{tabular}

(Continued)
Table 3 (Continued).

\begin{tabular}{|l|l|l|l|}
\hline & $\begin{array}{l}\text { Low } \\
\text { Adherence }\end{array}$ & $\begin{array}{l}\text { High } \\
\text { Adherence }\end{array}$ & P-value \\
\hline $\begin{array}{l}\text { IBD treatment } \\
\text { Monotherapy } \\
\text { Combination }\end{array}$ & $23(37 \%)$ & $39(63 \%)$ & 0.751 \\
\hline $\begin{array}{l}\text { Daily dose IBD medication } \\
\text { One }\end{array}$ & $15(34 \%)$ & $29(66 \%)$ & \\
More than one & $13(31 \%)$ & $29(69 \%)$ & \\
\hline MPR & $25(39 \%)$ & $39(61 \%)$ & \\
Low (<0.8) & & & 0.394 \\
High ( $\geq 0.8)$ & $31(91 \%)$ & $3(9 \%)$ & \\
\hline
\end{tabular}

as a significant predictor of low adherence in the previous research, ${ }^{22,23}$ and was also found to be relevant in our study which showed old age had high adherence. This could be explained by the fact that IBD primarily influenced younger persons who had a busy social life. In addition, younger patients were more rebellious. Furthermore, they had a more recent diagnosis and were less experienced with relapses and surgeries. Therefore, we suggested that special attention should be paid to adherence condition when dealing with younger patients. As WeChat was the main way for patients to obtain relevant information in China, young patients were encouraged to acquire the required disease knowledge on official accounts such as $\mathrm{CCCF}^{24}$ Clinicians should also give more attention to young patients and follow-up more frequently. And motivating them to help and communicate with each other was another way of improving adherence for young patients. In addition, IBD nurses were also an important factor in monitoring/improving adherence. They could enable patients to learn more about their disease, thus encouraging the patient's independence in managing their disease through guided self-management ability and increasing confidence, and directly improving adherence and benefitting their overall quality of life. ${ }^{25}$ In our study, we also found that patients who endured surgeries had high adherence, which was understandable. In addition, we also showed that patients who had small intestine lesions had lower adherence. One possible explanation was that lesions on small intestine were not as obvious as lesions on large intestine. Thus, patients had no symptoms or mild symptoms at early stage with small intestine. Hence, clinicians should pay more attention to the patients with small-bowel $\mathrm{CD}$ and take aggressive intervention 
Table 4 Predictive Factors for Adherence According to MPR (Univariate Analysis)

\begin{tabular}{|c|c|c|c|}
\hline & $\begin{array}{l}\text { Non- } \\
\text { Adherence }\end{array}$ & Adherence & $P$ value \\
\hline Patients & $34(32 \%)$ & $72(68 \%)$ & \\
\hline $\begin{array}{l}\text { Gender } \\
\text { Male } \\
\text { Female }\end{array}$ & $\begin{array}{l}24(33 \%) \\
10(30 \%)\end{array}$ & $\begin{array}{l}49(67 \%) \\
23(70 \%)\end{array}$ & 0.489 \\
\hline $\begin{array}{l}\text { Current age (years) } \\
\text { Age at diagnosis } \\
\text { Duration of disease (years) }\end{array}$ & $\begin{array}{l}34(29-37) \\
28 \pm 7 \\
4(2-6)\end{array}$ & $\begin{array}{l}33(29-39) \\
29 \pm 8 \\
4(3-7)\end{array}$ & $\begin{array}{l}0.596 \\
0.64 \\
0.92\end{array}$ \\
\hline $\begin{array}{l}\text { Currently Smoking, n (\%) } \\
\text { NO } \\
\text { YES }\end{array}$ & $\begin{array}{l}31(32 \%) \\
3(33 \%)\end{array}$ & $\begin{array}{l}66(68 \%) \\
6(67 \%)\end{array}$ & 0.598 \\
\hline $\begin{array}{l}\text { Abdominal surgery in the past, } \\
\text { n (\%) } \\
\text { NO } \\
\text { YES }\end{array}$ & $\begin{array}{l}25(35 \%) \\
9(26 \%)\end{array}$ & $\begin{array}{l}47(65 \%) \\
25(74 \%)\end{array}$ & 0.268 \\
\hline \multicolumn{4}{|l|}{ Disease localization, n (\%) } \\
\hline $\begin{array}{l}\text { Colon } \\
\text { NO } \\
\text { YES }\end{array}$ & $\begin{array}{l}12(40 \%) \\
22(29 \%)\end{array}$ & $\begin{array}{l}18(60 \%) \\
54(7 \mid \%)\end{array}$ & 0.192 \\
\hline $\begin{array}{l}\text { Small intestine } \\
\text { NO } \\
\text { YES }\end{array}$ & $\begin{array}{l}16(24 \%) \\
18(46 \%)\end{array}$ & $\begin{array}{l}51(76 \%) \\
21(54 \%)\end{array}$ & 0.016 \\
\hline $\begin{array}{l}\text { Both colon and small intestine } \\
\text { NO } \\
\text { YES }\end{array}$ & $\begin{array}{l}28(30 \%) \\
6(46 \%)\end{array}$ & $\begin{array}{l}65(70 \%) \\
7(54 \%)\end{array}$ & 0.197 \\
\hline $\begin{array}{l}\text { Marital status, n (\%) } \\
\text { Married } \\
\text { Non-Married }\end{array}$ & $\begin{array}{l}21(30 \%) \\
13(34 \%)\end{array}$ & $\begin{array}{l}47(70 \%) \\
25(66 \%)\end{array}$ & 0.443 \\
\hline $\begin{array}{l}\text { Highest level of education } \\
\text { completed, } n(\%) \\
\text { No } \\
\text { YES }\end{array}$ & $\begin{array}{l}14(27 \%) \\
20(37 \%)\end{array}$ & $\begin{array}{l}38(73 \%) \\
34(63 \%)\end{array}$ & 0.182 \\
\hline $\begin{array}{l}\text { Self-reported monthly income, } \\
\text { n (\%) } \\
\text { Under } 5000 ¥ / 5000-10,000 ¥ \\
\quad 10,000-20,000 ¥ / \text { above } \\
20,000 ¥\end{array}$ & $\begin{array}{l}16(30 \%) \\
18(35 \%)\end{array}$ & $\begin{array}{l}38(70 \%) \\
34(65 \%)\end{array}$ & 0.366 \\
\hline \multicolumn{4}{|l|}{ Medication use, $n$ (\%) } \\
\hline $\begin{array}{l}\text { 5-ASA use } \\
\text { NO } \\
\text { YES }\end{array}$ & $\begin{array}{l}17(35 \%) \\
17(30 \%)\end{array}$ & $\begin{array}{l}31(65 \%) \\
40(70 \%)\end{array}$ & 0.371 \\
\hline $\begin{array}{l}\text { Thiopurines } \\
\text { NO } \\
\text { YES }\end{array}$ & $\begin{array}{l}16(31 \%) \\
18(33 \%)\end{array}$ & $\begin{array}{l}36(69 \%) \\
36(67 \%)\end{array}$ & 0.47 \\
\hline
\end{tabular}

(Continued)
Table 4 (Continued).

\begin{tabular}{|l|l|l|l|}
\hline & $\begin{array}{l}\text { Non- } \\
\text { Adherence }\end{array}$ & Adherence & P value \\
\hline $\begin{array}{l}\text { IBD treatment } \\
\text { Monotherapy } \\
\text { Combination }\end{array}$ & $\begin{array}{l}21(34 \%) \\
13(30 \%)\end{array}$ & $\begin{array}{l}41(66 \%) \\
31(70 \%)\end{array}$ & 0.4 \\
\hline $\begin{array}{l}\text { Daily dose IBD medication } \\
\text { One } \\
\text { More than one }\end{array}$ & $\begin{array}{l}13(31 \%) \\
21(33 \%)\end{array}$ & $\begin{array}{l}29(69 \%) \\
43(67 \%)\end{array}$ & 0.507 \\
\hline $\begin{array}{l}\text { MMAS score } \\
\text { Low (<6) }\end{array}$ & $31(82 \%)$ & $\begin{array}{l}7(18 \%) \\
6(96 \%)\end{array}$ & 0.0001 \\
\hline High (6-8) & $3(4 \%)$ & $65 \%$ \\
\hline
\end{tabular}

Table 5 Multivariate Analysis in IBD Patients

\begin{tabular}{|l|l|l|}
\hline \multirow{2}{*}{} & \multicolumn{2}{|l|}{ Multivariate Analysis } \\
\cline { 2 - 3 } & Odds Ratio (95\% Cl) & P-value \\
\hline Current age & $1.08[1.02-1.13]$ & 0.0015 \\
Age at onset & $1.08[1.01-1.14]$ & 0.02 \\
Previous abdominal surgery & $3.18[2.09-4.27]$ & 0.04 \\
Small intestine lesion & $0.09[0.01-0.17]$ & 0.006 \\
\hline
\end{tabular}

during therapy. Besides, clinicians could clearly inform these patients the characteristics and prognosis of smallbowel Crohn's disease as well as the discordance between symptoms and objective measures of disease activity. Patients who were in clinical remission could have active evidence such as unhealed ulcers in bowels and elevated C-reactive protein level, while other patients with normal endoscopic finding and fecal cal-protectin level could still have persistent clinical symptoms. ${ }^{26,27}$ Currently, the treatto-target recommendations include endpoints of clinical (defined as resolution of abdominal pain) and endoscopic remission (defined as resolution of ulceration). ${ }^{26,28}$

Furthermore, we observed that the MMAS- 8 correlated significantly with MPR in 5-ASA group with a correlation coefficient of 0.4 , but no significant correlation was observed between MPR value and MMAS-8 score in azathioprine group. Therefore, in our study, MMAS-8 seemed to be a useful screening tool to predict IBD patients who took 5-ASA medication adherence behavior. Moreover, we also found that the values of MPR percentage were significantly correlated with MMAS- 8 unintentional adherence score with a correlation coefficient of $0.47(\mathrm{p}<0.001)$ in 5 -ASA group, but no significant 


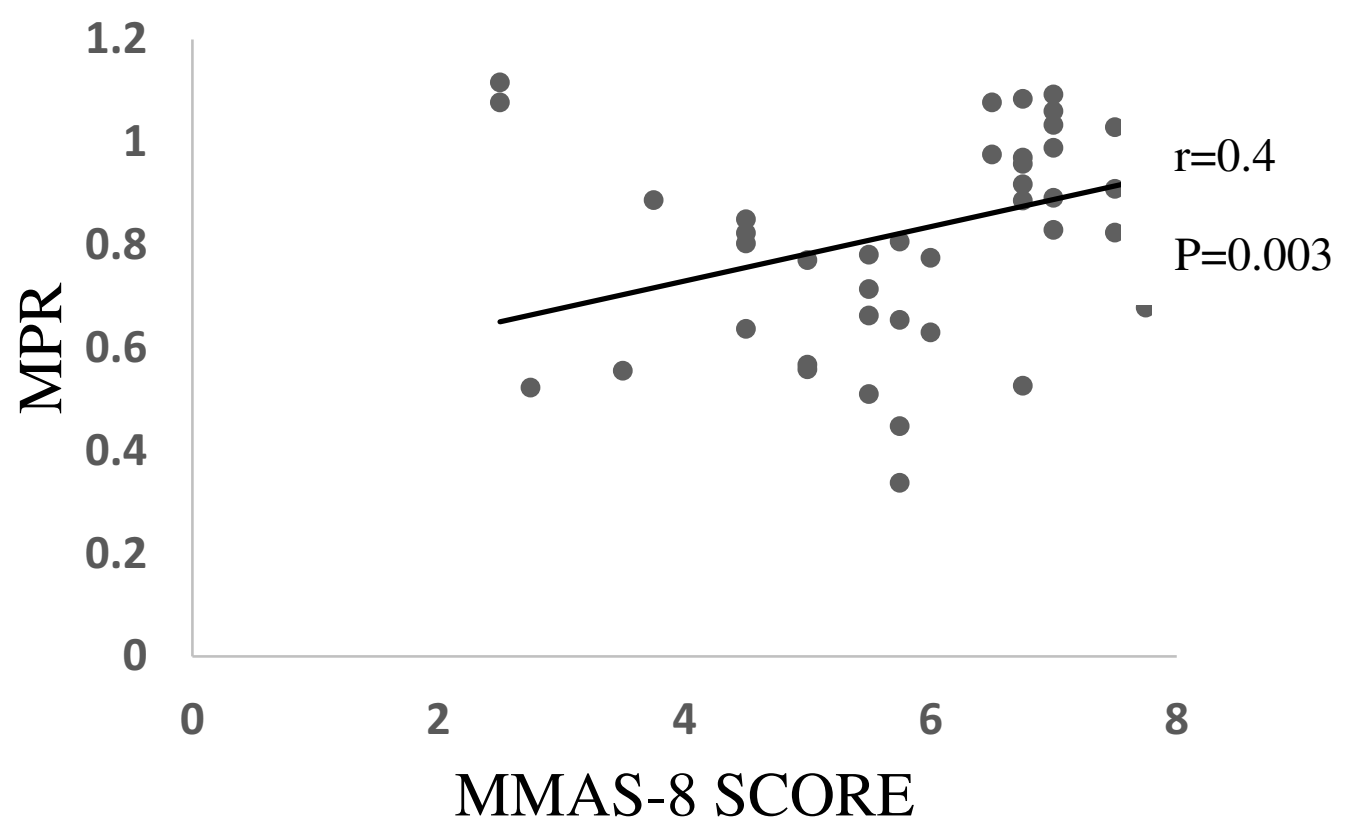

Figure I Correlation between MMAS-8 score and pharmacy refill behavior for 5-ASA.

correlation was observed between MPR value and MMAS-8 unintentional adherence score in azathioprine group. This may be partly due to "stockpiling behavior" in azathioprine group. Most of the patients prefer to stocking up excessive medicines in case of lacking thus influenced the validity of MPR. Hence, in our study, MMAS-8 seemed to be a useful tool to assess patients' reasons for non-adherence and differentiate between intentional and unintentional non-adherence in 5-ASA group. This observation was consistent with another research that investigated the correlation between the MMAS- 8 tool and MPR among 150 IBD patients receiving different therapies, including 5-ASA, steroids, immunomodulators, and antiTNF agents. ${ }^{15}$ The MMAS-8 correlated significantly with MPR in immunomodulators group with a correlation coefficient of 0.26 , but not in 5-ASA group and anti-TNF group. ${ }^{15}$ However, other studies that excluded anti-TNF group found a significant association between the MMAS8 tool and MPR. ${ }^{29}$ These differences may be explained by the fact that the MMAS- 8 tool was originally developed in patients on daily medication.

The strengths of our study include a diverse population with variable social demographic profile, disease activity and medication history. In addition, we combined MMAS- 8 tool and pharmacy refill data for measuring adherence in order to improve accuracy. Furthermore, as far as we know, our study firstly reported on adherence to IBD maintenance medication in Chinese patients. The limitations of our study need to be addressed as well. First, the sample size was small. And the survey was conducted with patients who attended a tertiary referral practice, which might not represent patients in the community. Second, the Hawthorne effect, which means that patients might improve their medication-taking behavior in response to their awareness of being observed, could not be completely excluded. ${ }^{30}$ Third, previous researches observed that adherent patients had a higher belief of necessity for therapy and fewer doubts about the efficacy of IBD medication. ${ }^{31,32}$ The influence of patients' beliefs on medication adherence was not taken into account in our study.

In summary, we had identified factors that might influence on adherence in Chinese IBD patients. We found that predictors of low adherence were young age, lesions on small intestine, whereas previous abdominal surgery was a protective factor. Therefore, clinicians should pay more attention and closely follow-up younger patients and smallbowel Crohn's disease patients to improve their adherence. In addition, the MMAS-8 had shown to be a convenient tool to measure adherence in chronic disease patients, which was simple, practical and patient-friendly questionnaire that could be done through the internet or mobile. The use of pharmacy refills seemed to be a gold standard; however, it is complicated and time consuming if the patient got refills from multiple sources. In our study, we also had demonstrated that the MMAS-8 tool correlated well with MPR, which could identify low adherers in Chinese IBD patients consistent with 


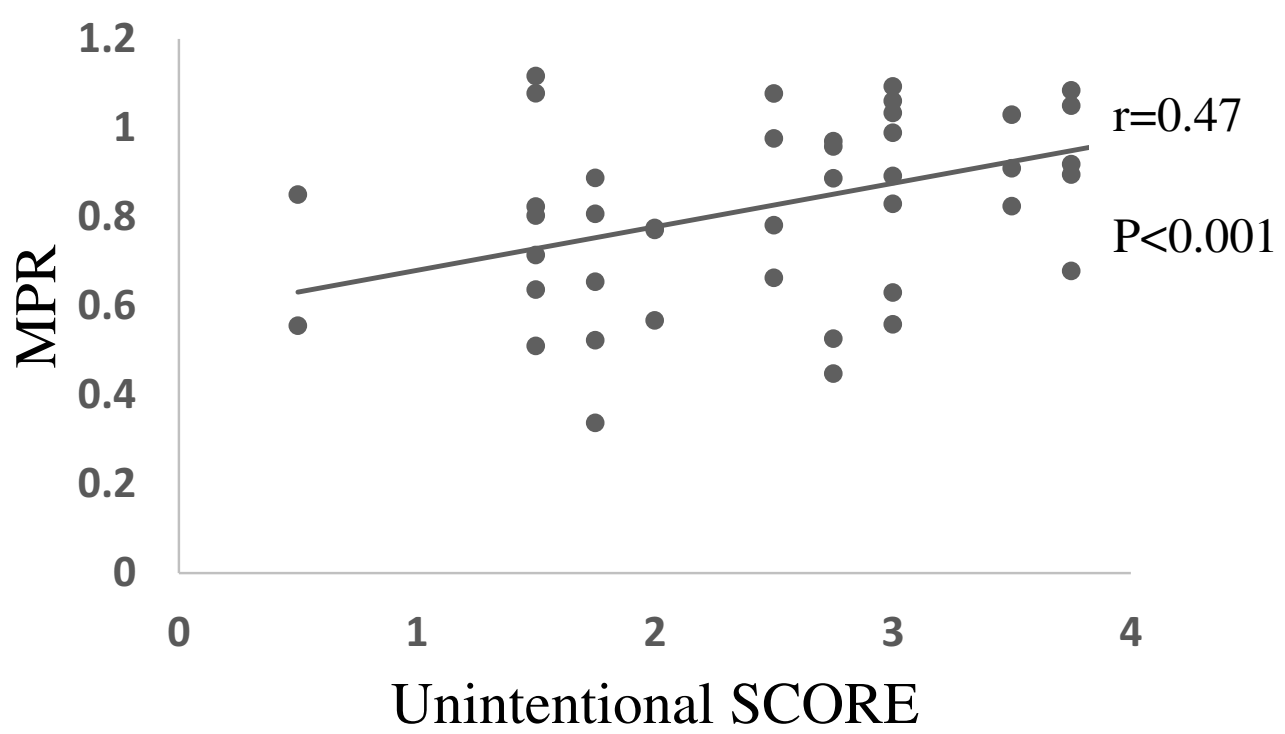

Figure 2 Correlation between MMAS-8 unintentional score and pharmacy refill behavior for 5-ASA.

previous work, but it appeared to be limited for specific drug classes like 5-ASA. More work should be done to legitimize this tool in everyday clinical practice.

\section{Highlights}

- Poor medication adherence in inflammatory bowel disease (IBD) had a negative impact on disease outcomes.

- Predictors of low adherence were young age, lesions on small intestine, whereas previous abdominal surgery was a protective factor.

- MMAS-8 scale was a valid instrument for assessing 5-ASA adherence in IBD patients.

\section{Abbreviations}

IBD, inflammatory bowel disease; CD, Crohn's disease 16; UC, ulcerative colitis; MMAS, Morisky Medication Adherence Scale; MPR, Medication Possession Ratio; 5-ASA, 5-aminosalicylic acid; CCCF, The China Crohn's \& Colitis Foundation 17; HBI, Harvey- Bradshaw Index; P-SSCAI, Patient-based Simple Clinical Colitis Activity Index.

\section{Funding}

This work was supported by research grants from Zhejiang Provincial National Science Foundation of China (grant number LY20H160031) and Intestinal Barrier Research Foundation of Lijieshou (grant number LJS-201703) to CPZ. This work was also supported by Ferring company, Shanghai, China.

\section{Disclosure}

The authors declare no conflicts of interest in this work.

\section{References}

1. Ng SC, Tang W, Ching JY, et al. Incidence and phenotype of inflammatory bowel disease based on results from the Asia-Pacific Crohn's and colitis epidemiology study. Gastroenterology. 2013;145(1):158165.e2. doi:10.1053/j.gastro.2013.04.007

2. Gomollón F, Dignass A, Annese V, et al. 3rd European evidence-based consensus on the diagnosis and management of Crohn's disease 2016: part 1: diagnosis and medical management. J Crohn's Colitis. 2017;11 (1):3-25. doi:10.1093/ecco-jcc/jjw168

3. Kane SV, Chao J, Mulani PM. Adherence to infliximab maintenance therapy and health care utilization and costs by Crohn's disease patients. Adv Ther. 2009;26(10):936-946. doi:10.1007/s12325-009-0069-7

4. Higgins PDR, Rubin DT, Kaulback K, Schoenfield PS, Kane SV. Systematic review: impact of non-adherence to 5-aminosalicylic acid products on the frequency and cost of ulcerative colitis flares. Aliment Pharmacol Ther. 2009;29(3):247-257. doi:10.1111/j.1365-2036.2008.03865.x

5. Tomar SK, Kedia S, Singh N, et al. Higher education, professional occupation, and upper socioeconomic status are associated with lower adherence to medications in patients with inflammatory bowel disease. J Gastroenterol Hepatol. 2019;3:302-309.

6. Jackson CA, Clatworthy J, Robinson A, Horne R. Factors associated with non-adherence to oral medication for inflammatory bowel disease: a systematic review. Am J Gastroenterol. 2010;105(3):525-539. doi:10.1038/ajg.2009.685

7. Campos S, Portela F, Sousa P, Sofia C. Inflammatory bowel disease: adherence to immunomodulators in a biological therapy era. Eur J Gastroenterol Hepatol. 2016;28(11):1313-1319. doi:10.1097/ MEG.0000000000000704

8. Coenen S, Weyts E, Ballet V, et al. Identifying predictors of low adherence in patients with inflammatory bowel disease. Eur $J$ Gastroenterol Hepatol. 2016;28(5):503-507. doi:10.1097/MEG.00 00000000000570

9. Eindor-abarbanel A, Naftali T, Ruhimovich N, et al. Revealing the puzzle of nonadherence in IBD - assembling the pieces. Inflamm Bowel Dis. 2018;24(6):1352-1360. doi:10.1093/ibd/izy013 
10. Herman ML, Kane SV. Treatment nonadherence in in flammatory bowel disease: identifi cation, scope, and management strategies. Inflamm Bowel Dis. 2015;21:2979-2984. doi:10.1097/MIB.00000 00000000581

11. Trindade AJ, Ehrlich A, Kornbluth A, Ullman TA. Are your patients taking their medicine? Validation of a new adherence scale in patients with inflammatory bowel disease and comparison with physician perception of adherence. Inflamm Bowel Dis. 2011;17(2):599-604. doi:10.1002/ibd.21310

12. Morisky DE, Ang A, Krousel-wood M, Ward HJ. Predictive validity of a medication adherence measure in an outpatient setting. J Clin Hypertens. 2008;10(5):348-354. doi:10.1111/j.1751-7176.2008.075 72.x

13. Krousel-Wood M, Islam T, Webber LS, Re RN, Morisky DE, Muntner P. New medication adherence scale versus pharmacy fill rates in seniors with hypertension. Am J Manag Care. 2009;15 (1):59-66.

14. Voils CI, Hoyle RH, Thorpe CT, MacIejewski ML, Yancy Jr. WS. Improving the measurement of self-reported medication nonadherence. J Clin Epidemiol. 2011;64(3):250-254. doi:10.1016/ j.jclinepi.2010.07.014

15. Hess LM, Raebel MA, Conner DA, Malone DC. Measurement of adherence in pharmacy administrative databases: a proposal for standard definitions and preferred measures. Ann Pharmacother. 2006;40 (7-8):1280-1288. doi:10.1345/aph.1H018

16. BennebroekEvertsz' F, Nieuwkerk PT, Stokkers PCF, et al. The patient simple clinical colitis activity index (P-SCCAI) can detect ulcerative colitis (UC) disease activity in remission: a comparison of the P-SCCAI with clinician-based SCCAI and biological markers. J Crohn's Colitis. 2013;7:890-900. doi:10.1016/j.crohns.2012.11.007

17. Harvey RF, Bradshaw JM. A simple index of Crohn's-disease activity. Lancet. 1980;315(8167):514. doi:10.1016/S0140-6736(80)92767-1

18. van der Have M, Oldenburg B, Kaptein AA, et al. Non-adherence to anti-TNF therapy is associated with illness perceptions and clinical outcomes in outpatients with inflammatory bowel disease: results from a prospective multicentre study. J Crohn's Colitis. 2016;10 (5):549-555. doi:10.1093/ecco-jcc/jjw002

19. Bucci C, Zingone F, Tammaro S, Iovino P, Santonicola A. Factors predicting the adherence to the therapy of Italian IBD patients. Gastroenterol Res Pract. 2017;2017:6719345. doi:10.1155/2017/ 6719345

20. Robinson A. Review article: improving adherence to medication in patients with inflammatory bowel disease. Aliment Pharmacol Ther. 2008;27(Suppl 1):9-14. doi:10.1111/j.1365-2036.2008.03604.x
21. Bruna-Barranco I, Lué A, Gargallo-Puyuelo CJ, et al. Young age and tobacco use are predictors of lower medication adherence in inflammatory bowel disease. Eur J Gastroenterol Hepatol. 2019;31 (8):948-953. doi:10.1097/MEG.0000000000001436

22. Červený P, Bortlík M, Kuběna A, Vlček J, Lakatos PL, Lukáš M. Nonadherence in inflammatory bowel disease: results of factor analysis. Inflamm Bowel Dis. 2007;13(10):1244-1249. doi:10.1002/ ibd.20189

23. D'Incà R, Bertomoro $\mathrm{P}$, Mazzocco $\mathrm{K}$, Vettorato MG, Rumiati R, Sturniolo GC. Risk factors for non-adherence to medication in inflammatory bowel disease patients. Aliment Pharmacol Ther. 2008;27(2):166-172. doi:10.1111/j.1365-2036.2007.03555.x

24. Yu Q, Xu L, Li L, et al. Internet and WeChat used by patients with Crohn's disease in China: a multi-center questionnaire survey. BMC Gastroenterol. 2019;19(1):97. doi:10.1186/s12876-019-1011-3

25. Kemp K, Dibley L, Chauhan U, et al. Second N-ECCO consensus statements on the European nursing roles in caring for patients with Crohn's disease or ulcerative colitis. J Crohn's Colitis. 2018;12 (7):760-776. doi:10.1093/ecco-jcc/jjy020

26. Colombel JF, D'haens G, Lee WJ, Petersson J, Panaccione R. Outcomes and strategies to support a treat-to-target approach in inflammatory bowel disease: a systematic review. J Crohns Colitis. 2020;14(2):254-266. doi:10.1093/ecco-jcc/jjz131

27. Peyrin-Biroulet L, Reinisch W, Colombel JF, et al. Clinical disease activity, C-reactive protein normalisation and mucosal healing in Crohn's disease in the SONIC trial. Gut. 2014;63(1):88-95. doi:10.1136/gutjnl-2013-304984

28. Agrawal M, Colombel JF. Treat-to-target in inflammatory bowel diseases, what is the target and how do we treat? Gastrointest Endosc Clin N Am. 2019;29(3):421-436. doi:10.1016/j.giec.2019.02.004

29. Kane S, Becker B, Harmsen WS, Kurian A, Morisky DE, Zinsmeister AR. Use of a screening tool to determine nonadherent behavior in inflammatory bowel disease. Am J Gastroenterol. 2012;107:154-160. doi:10.1038/ajg.2011.317

30. McCarney R, Warner J, Iliffe S, van Haselen R, Griffin M, Fisher P. The Hawthorne effect: a randomised, controlled trial. BMC Med Res Methodol. 2007;7:30. doi:10.1186/1471-2288-7-30

31. Sewitch MJ, Abrahamowicz M, Barkun A, et al. Patient nonadherence to medication in inflammatory bowel disease. Am J Gastroenterol. 2003;98(7):1535-1544. doi:10.1111/j.1572-0241.2003.07522.x

32. Selinger CP, Eaden J, Brian Jones D, et al. Modifiable factors associated with nonadherence to maintenance medication for inflammatory bowel disease. Inflamm Bowel Dis. 2013;19(10):2199-2206. doi:10.1097/MIB.0b013e31829ed8a6
Patient Preference and Adherence

\section{Publish your work in this journal}

Patient Preference and Adherence is an international, peer-reviewed, open access journal that focuses on the growing importance of patient preference and adherence throughout the therapeutic continuum. Patient satisfaction, acceptability, quality of life, compliance, persistence and their role in developing new therapeutic modalities and compounds to optimize clinical outcomes for existing disease states are major areas of interest for the journal. This journal has been accepted for indexing on PubMed Central. The manuscript management system is completely online and includes a very quick and fair peer-review system, which is all easy to use. Visit http:// www.dovepress.com/testimonials.php to read real quotes from published authors. 